\title{
The Prevalence and Incidence of Dementia with Lewy Bodies: a Systematic Review
}

\author{
David B. Hogan, Kirsten M. Fiest, Jodie I. Roberts, Colleen J. Maxwell, \\ Jonathan Dykeman, Tamara Pringsheim, Thomas Steeves, Eric E. Smith, \\ Dawn Pearson, Nathalie Jetté
}

\begin{abstract}
Background: Population-based prevalence and incidence studies are essential for understanding the societal burden of dementia with Lewy bodies (DLB). Methods: The MEDLINE and EMBASE databases were searched to identify publications addressing the incidence and/or prevalence of DLB. References of included articles and prior systematic reviews were searched for additional studies. Two reviewers screened all abstracts and full-text reviews, abstracted data and performed quality assessments. Results: Twenty-two studies were included. Incidence rates ranged from 0.5 to 1.6 per 1000 person-years. DLB accounted for 3.2-7.1\% of all dementia cases in the incidence studies. Point and period prevalence estimates ranged from 0.02 to 63.5 per 1000 persons. Increasing prevalence estimates were reported with increasing age. DLB accounted for from 0.3 to $24.4 \%$ of all cases of dementia in the prevalence studies. Conclusions: DLB becomes more common with increasing age and accounts for about $5 \%$ of all dementia cases in older populations.
\end{abstract}

RÉSUMÉ: Prévalence et incidence de la démence à corps de Lewy : revue systématique du sujet. Contexte: Les études de population sur sa prévalence et son incidence sont essentielles à la compréhension du fardeau social de la démence à corps de Lewy (DCL). Méthodologie: Nous avons identifié les publications sur l'incidence et/ou la prévalence de la DCL dans les bases de données MEDLINE et EMBASE. Nous avons recherché des études supplémentaires dans les articles et les revues systématiques antérieures cités à titre de références. Deux évaluateurs ont examiné tous les résumés et les évaluations de textes intégraux et l'extraction des données, et ils ont évalué la qualité des publications. Résultats: Vingt-deux études ont été incluses. Les taux d'incidence allaient de 0,5 à 1,6 par 1000 personnes-années. La DCL constituait 3,2 à 7,1\% de tous les cas de démence dans les études sur l'incidence. Les estimés de prévalence ponctuelle et par période allaient de 0,02 à 63,5 par 1000 personnes. Des taux croissants de prévalence étaient rapportés en association avec l'augmentation de l'âge des sujets. La DCL était responsable de 0,3 à $24,4 \%$ de tous les cas de démence dans les études de prévalence. Conclusions: La DCL est plus fréquente avec l'âge et constitue environ 5\% de tous les cas de démence dans les populations plus âgées.

Keywords: Dementia with Lewy bodies, Lewy body disease, incidence, prevalence, epidemiology, systematic review

doi:10.1017/cjn.2016.2

Can J Neurol Sci. 2016; 43: S83-S95

\section{INTRODUCTION}

Dementia with Lewy bodies (DLB) is one of the commonest neurodegenerative dementias encountered in older patients. ${ }^{1}$ While recent clinic-based studies suggest that it accounts for up to $20 \%$ of all dementia cases, ${ }^{1-5}$ this may represent an overestimate because of selection or referral bias. ${ }^{6}$ Population-based estimates of attributable risk derived from autopsy studies suggest that Lewy body disease accounts for 3 to $10 \%$ of the burden of dementia in older populations. ${ }^{7,8}$

Building on clinicopathological work, ${ }^{9-11}$ consensus criteria for the diagnosis of DLB have been developed. ${ }^{12-14}$ Their central feature is the presence of progressive cognitive decline of sufficient magnitude to interfere with normal social or occupational functioning. During the early stages of the illness, deficits in attention, executive function and visuospatial ability are often more striking than those with memory. The core clinical features of DLB are fluctuations in cognition with pronounced variations in attention and alertness, recurrent detailed visual hallucinations and spontaneous parkinsonism. The latest revision ${ }^{14}$ added three suggestive features (neuroleptic sensitivity, reduced basal ganglia dopamine uptake on single-photon emission computed tomography [SPECT] or PET imaging, REM sleep behaviour disorder). Probable DLB is diagnosed if two core features or one core and at

From the Brenda Strafford Chair in Geriatric Medicine, University of Calgary, Calgary, Alberta, Canada (DBH); the Hotchkiss Brain Institute, University of Calgary, Calgary, Alberta, Canada (DBH, KMF, JIR, JD, TP, EES, NJ); Department of Medicine, Cumming School of Medicine, University of Calgary, Calgary, Alberta, Canada (DBH); Department of Community Health Sciences, University of Calgary, Calgary, Alberta, Canada (KMF, JIR, CJM, JD, TP, EES, NJ); Department of Clinical Neurosciences, University of Calgary, Calgary, Alberta, Canada (KMF, JIR, JD, TP, EES, DP, NJ); the Institute for Public Health, University of Calgary, Calgary, Alberta, Canada (KMF, JIR, JD, TP, NJ); the Institute for Clinical Evaluative Sciences, Toronto, Ontario, Canada (CJM); the Schools of Pharmacy and Public Health \& Health Systems, University of Waterloo, Waterloo, Ontario, Canada (CJM); Department of Medicine, University of Toronto, Toronto, Ontario, Canada (TS).

Received October 14, 2015. Final Revisions Submitted December 21, 2015. Correspondence to: Nathalie Jetté, Foothills Medical Center, Department of Clinical Neurosciences, 1403 29th Street NW, Calgary, Alberta T2N 4N1, Canada.

Email: Nathalie.jette@albertahealthservices.ca 
least one suggestive feature are present. Possible DLB requires the presence of either one core or suggestive feature.

Population-based prevalence and incidence studies are essential for understanding the societal burden of DLB and planning for the range of healthcare services needed for these individuals. In this paper, we report on a systematic review of population-based prevalence and/or incidence studies of DLB. This is a relatively understudied area, with only two prior systematic reviews on the epidemiology of DLB published. ${ }^{15,16}$ Since the appearance of the first one in $2005,{ }^{15}$ awareness of DLB has increased with both revised diagnostic criteria ${ }^{14}$ and additional studies reporting primary data published. The earlier systematic review ${ }^{15}$ was based on seven studies compared to the 22 that we included. The second systematic review ${ }^{16}$ is more current, but there were significant differences in the methodology used by the authors compared to our work. For example, their literature search was limited to one database (PubMed), and different criteria were utilized for the selection of articles. Of the 32 population-based studies included in either systematic review, only 11 [34.4\%] were common to both. As our systematic review includes studies not incorporated in the previously published systematic reviews and utilizes different study methods, we feel it offers an additional perspective for the literature on this topic.

\section{Methods}

This is one of a series of systematic reviews on the incidence and prevalence of priority neurological conditions identified by the Public Health Agency of Canada and Neurological Health Charities Canada as part of the National Population Health Study of Neurological Conditions. These conditions included dementia.

\section{Search Strategy and Study Selection}

The MEDLINE and EMBASE databases were searched using terms and approaches developed by the authors in consultation with an academic librarian to identify publications dealing with the incidence and/or prevalence of dementia and the commoner dementia aetiologies in middle-aged and older adults (i.e., Alzheimer's disease, vascular dementia, frontotemporal dementia, DLB) (see Appendix A for detailed search terms). Population-based studies that reported on the incidence and/or prevalence of dementia and the commoner aetiologies were included provided they: (1) were published in English or French; (2) reported primary or original data; and (3) had a publication date of 2000 or later (international studies) or a publication date of 1985 or later (Canadian studies). The 2000-or-later publication date was used to avoid overlap with previous systematic reviews of the epidemiology of dementia, while the earlier date for Canadian studies was chosen to ensure that all relevant national reports were included (note: no Canadian studies on DLB were found).

Two reviewers independently screened all retrieved abstracts and performed full-text reviews on selected papers. Disagreements were resolved by consensus with the option of involving a third reviewer if one could not be reached (which was never required). The reviewers also searched the references of included articles and prior systematic reviews in order to detect additional eligible studies. Only studies that reported on the incidence and/or prevalence of DLB (or provided the necessary information to calculate an estimate) were included.

As more than a year elapsed between the initial literature search and completion of data abstraction, the EMBASE and MEDLINE database searches were updated (July of 2012). Search terms dealing with dementia aetiologies other than DLB were removed (see Appendix B for detailed search terms); otherwise, the methodology employed (including inclusion criteria and review procedures) was identical to that used in the original search.

\section{Data Abstraction and Study Quality}

The two reviewers independently abstracted data using a standard form. Overall prevalence and/or incidence values for DLB were recorded along with age- and sex-stratified data when available. Information regarding study location, age range of the study population (i.e., $<65,60+, 65+, 70+, 75+, 81+$ ), case ascertainment methods, diagnostic criteria and definition of DLB were collected. The reviewers independently completed a quality assessment for each included study using an instrument specifically designed for this series of systematic reviews. Based on prior recommendations, ${ }^{17,18}$ studies were assessed on eight characteristics (an affirmative response was given one point, while all others were scored zero): (1) target population was clearly defined; (2) cases were ascertained by either a survey of the entire population or probability sampling; (3) the response rate was $>70 \%$; (4) nonresponders were described; (5) the sample was representative of the population; (6) data collection methods were standardized; (7) validated diagnostic criteria were used; and (8) presentation of incidence and/or prevalence estimates was reported with confidence intervals or by subgroup. Possible scores ranged from 0 to 8 , with higher ones indicating a better quality assessment. For studies where the estimates were based on information provided by practitioners and/or health administration data, reviewers responded in the affirmative for the third, fifth and sixth points, and "not applicable" for the fourth. Any discrepancies were resolved by consensus or involvement of a third reviewer, if necessary.

\section{Data Analysis}

A pooled meta-analysis was not done due to significant between-study heterogeneity and the small sample size. Forest plots presenting the distribution of study estimates were produced. All statistical analyses were carried out in $\mathrm{R}$ version 2.14 , and the Meta package was employed to produce forest plots. Because of higher diagnostic specificity, ${ }^{12}$ the most restrictive definition for DLB that could be obtained from the reviewed papers (e.g., probable DLB rather than possible DLB) was used in our primary analyses of incidence and/or prevalence. Depending on study methodology, incidence proportion (or cumulative incidence, the proportion of the population at risk who develop DLB within a defined period), incidence rate (the rate at which new cases of DLB occur in a population during a defined period), period prevalence (total number of persons known to have DLB during a specified period) and point prevalence (the number of persons with DLB at a specified point in time) were provided. 


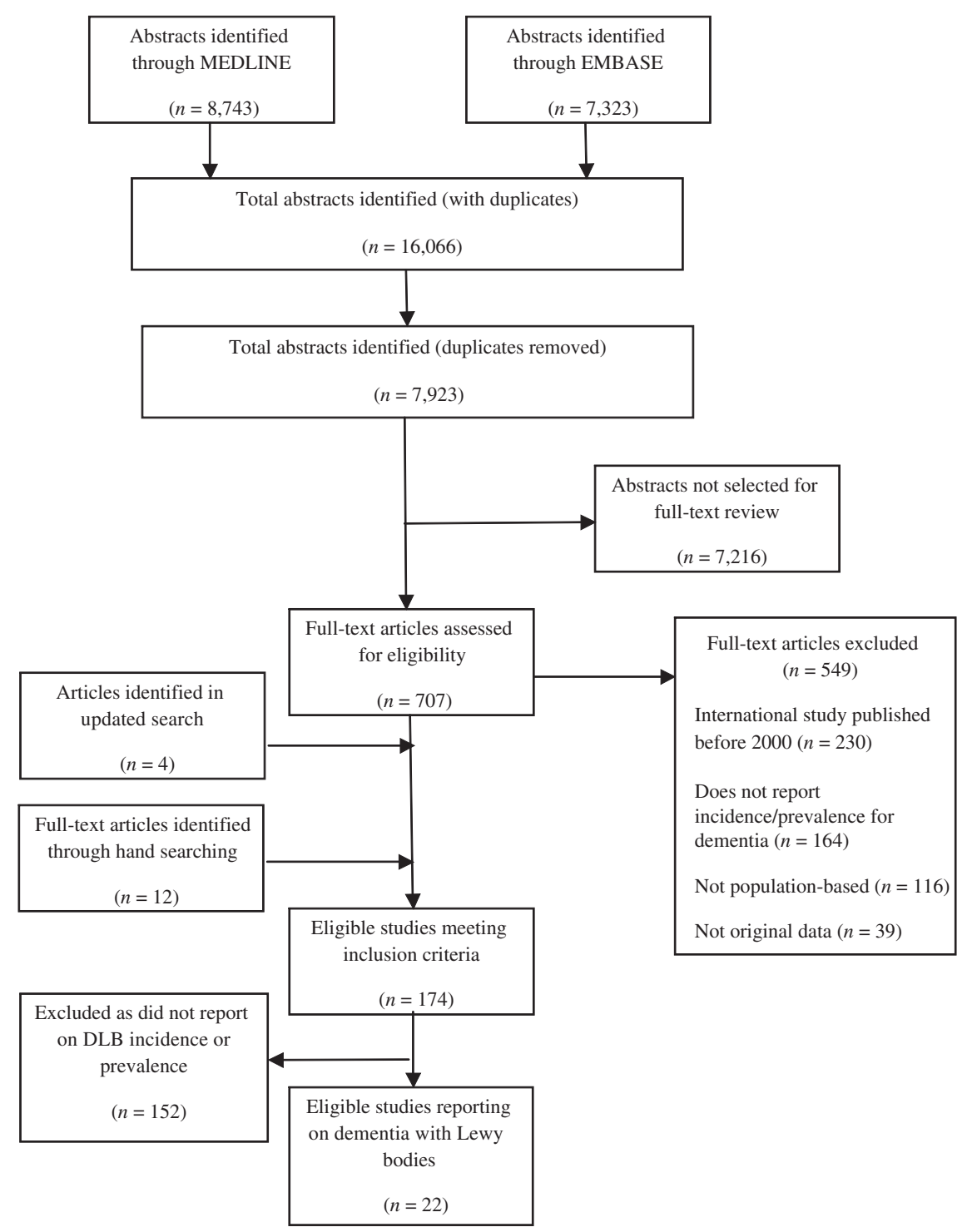

Figure 1: Flowchart of studies.

\section{RESULTS}

\section{Search Strategy}

In the initial search, 7923 unique references were identified on MEDLINE and/or EMBASE (see Figure 1). A total of 707 articles were selected for full-text review. Of these, 230 were excluded on the basis of publication date, 164 for not reporting on the incidence and/or prevalence of dementia, 116 as they were not population-based and 39 because they did not report original data. The updated MEDLINE and EMBASE search yielded a further 334 references. Eleven were selected for full-text review, and four met our inclusion criteria. An additional 12 articles were identified from the references of selected articles. A total of 174 articles met our inclusion criteria for dementia. Twenty-two of them reported on DLB and were included in our systematic review. ${ }^{19-40}$ Of these papers, four focused solely on DLB, ${ }^{19,34,35,40}$ while 18 dealt with some other aspect of the epidemiology of dementia but included data on DLB. ${ }^{20-33,36-39}$

\section{Selected Studies}

Seventeen of the studies reported on prevalence $e^{19-29,35-40}$ and five on incidence. ${ }^{30-34}$ The mean quality score was 5.9 (standard deviation $[S D] 1.3$; range 4-8). Nineteen received scores of $5+$. Please see Table 3 for the quality scores of individual studies.

Please refer to Tables 1 and 2 for a summary of study characteristics. Eight originated from the Western Pacific (Australia 1, Japan 6, Korea 1), 7 from Europe (Finland 1, France 1, Italy 1, Spain 1, Turkey 1, United Kingdom 2), 6 from the Americas (Brazil 3, Cuba 1, United States 2) and 1 from Southeast Asia (Sri Lanka). Most $(n=21)$ of the studies reported on the incidence and/or prevalence in a defined geographic area. The exception examined prevalence among residents of 22 randomly selected assisted-living facilities in the city of Baltimore and several Maryland counties. ${ }^{36}$ Eight studies provided information on sex, with four reporting that it was more common among women $^{24,29,34,35}$ and four among men. ${ }^{33,37,39,40}$ Two studies 
Table 1: Studies reporting on the prevalence of dementia with Lewy bodies

\begin{tabular}{|c|c|c|c|c|c|c|c|}
\hline Author (date) & $\begin{array}{l}\text { Age range } \\
\text { (mean age) }\end{array}$ & $\begin{array}{l}\text { WHO geographic } \\
\text { region, country, \& } \\
\text { community }\end{array}$ & $\begin{array}{l}\text { Sample source and potential case } \\
\text { ascertainment process }\end{array}$ & $\begin{array}{l}\text { Method used to make } \\
\text { diagnosis }\end{array}$ & Diagnostic criteria used & $\begin{array}{l}\text { Date study } \\
\text { done }\end{array}$ & $\begin{array}{l}\text { Overall prevalence estimate } \\
\text { (\# cases) \& comments }\end{array}$ \\
\hline \multicolumn{8}{|c|}{ Community only (listed alphabetically) } \\
\hline Bennett $(2003)^{19}$ & $\begin{array}{l}81+(87.9 \text { for } \\
\text { probable } \\
\text { DLB cases })\end{array}$ & $\begin{array}{l}\text { Western Pacific } \\
\text { Australia } \\
\text { Sydney }\end{array}$ & $\begin{array}{l}\text { Wave } 3 \text { assessment of a random sample } \\
\text { of community dwellers } 75+\text { when } \\
\text { initially recruited ( } 299 \text { of initial } 630 \\
\text { participants); in-home assessment } \\
\text { including examination \& cognitive } \\
\text { testing of all participants }\end{array}$ & $\begin{array}{l}\text { Diagnoses made by } \\
\text { non-examining neurologist/ } \\
\text { geriatrician based on } \\
\text { assessment data }\end{array}$ & $\begin{array}{l}\text { Dementia: CDR* score of } \\
\quad>0.5^{41} \\
\text { DLB: McKeith }(1996)^{12} \\
\text { for probable DLB }\end{array}$ & 1997-1999 & $\begin{array}{l}63.5 \text { per } 1000 \text { (19 cases) } \\
\text { Comment: Additional } 34 \text { cases } \\
\text { of possible DLB; probable } \\
\text { DLB accounted for } 24.4 \% \text { of } \\
\text { dementia cases (possible } \\
\text { DLB } 43.6 \% \text { ) }\end{array}$ \\
\hline Bottino $(2008)^{20}$ & $60+(71.4)$ & $\begin{array}{l}\text { Americas } \\
\text { Brazil } \\
\text { Sao Paulo }\end{array}$ & $\begin{array}{l}\text { Cluster random sample stratified by } \\
\text { socioeconomic class of city residents } \\
60+; 2 \text { stages (screening \& diagnostic } \\
\text { evaluation if screened positive) }\end{array}$ & $\begin{array}{l}\text { Consensus diagnoses based on } \\
\text { clinical assessment (all that } \\
\text { was available on participants } \\
\text { seen at home), laboratory } \\
\text { investigations, \& } \\
\text { neuroimaging }\end{array}$ & $\begin{array}{l}\text { Dementia: DSM-IV } \mathrm{V}^{\dagger 40} \\
\text { DLB: not stated }\end{array}$ & 2000 & $\begin{array}{l}0.6 \text { per } 1000(1 \text { case }) \\
\text { Comment: Additional case of } \\
\text { PDD }^{\ddagger} \text { DLB accounted for } \\
0.9 \% \text { of dementia cases }\end{array}$ \\
\hline $\begin{array}{l}\text { de Jesús Llibre } \\
\qquad(2009)^{21}\end{array}$ & $65+(74.6)$ & $\begin{array}{l}\text { Americas } \\
\text { Cuba } \\
\text { Playa Municipality }\end{array}$ & $\begin{array}{l}\text { All municipality residents } 65+; 2 \text { stages } \\
\text { (initial assessment followed by } \\
\text { detailed evaluation of } 20 \% \text { of those } \\
\text { felt to be normal \& all those with } \\
\text { suspected cognitive concerns) }\end{array}$ & $\begin{array}{l}\text { Consensus diagnoses based on } \\
\text { clinical assessment, } \\
\text { caregiver interview, } \\
\text { laboratory investigations, } \\
\text { and neuroimaging }\end{array}$ & $\begin{array}{l}\text { Dementia: DSM-IV }{ }^{40} \\
\text { DLB: McKeith }(1992)^{10} \\
\text { for senile dementia of } \\
\text { Lewy body type }\end{array}$ & 2003 & $\begin{array}{l}0.3 \text { per } 1000 \text { ( } 5 \text { cases }) \\
\text { Comment: Additional } 33 \text { cases } \\
\text { of PDD; DLB accounted for } \\
0.3 \% \text { of dementia cases }\end{array}$ \\
\hline de Silva $(2003)^{22}$ & $65+(69.4)$ & $\begin{array}{l}\text { Southeast Asia } \\
\text { Sri Lanka } \\
\text { Ragama }\end{array}$ & $\begin{array}{l}\text { Random sample of town residents } 65+ \\
\text { from } 4 \text { primary health care divisions; } \\
2 \text { stages (screening \& diagnostic } \\
\text { evaluation if screen positive) }\end{array}$ & $\begin{array}{l}\text { Based on clinical assessment, } \\
\text { caregiver interview, } \\
\text { laboratory investigations and } \\
\text { neuroimaging but not clear } \\
\text { who made final diagnosis }\end{array}$ & $\begin{array}{l}\text { Dementia: DSM-IV }{ }^{40} \\
\text { DLB: McKeith }(1996)^{12} \\
\text { for probable DLB }\end{array}$ & 2000 & $\begin{array}{l}1.4 \text { per } 1000 \text { ( } 1 \text { case }) \\
\text { Comment: DLB accounted for } \\
3.6 \% \text { of dementia cases }\end{array}$ \\
\hline Gurvit $(2008)^{24}$ & $70+(74.9)$ & $\begin{array}{l}\text { Europe } \\
\text { Turkey } \\
\text { Istanbul (Kadıköy } \\
\quad \text { district) }\end{array}$ & $\begin{array}{l}\text { Random sample of community- } \\
\text { dwelling residents } 70+(95.5 \% \\
\text { consented); } 2 \text { stages (screening } \\
\text { followed by diagnostic assessment on } \\
\text { those failing cognitive screen plus } 9 \% \\
\text { of those who did not) }\end{array}$ & $\begin{array}{l}\text { Diagnosis made at consensus } \\
\text { conference with the team } \\
\text { leader (physician) after a } \\
\text { thorough chart review }\end{array}$ & $\begin{array}{l}\text { Dementia: DSM-III }{ }^{39} \\
\text { DLB: McKeith }(1996)^{12} \\
\text { but not stated if possible } \\
\text { and/or probable }\end{array}$ & Not stated & $\begin{array}{l}8.8 \text { per } 1000 \text { ( } 9 \text { cases }) \\
\text { Comment: DLB accounted for } \\
9.7 \% \text { of dementia cases }\end{array}$ \\
\hline Herrera $(2002)^{26}$ & $65+$ & $\begin{array}{l}\text { Americas } \\
\text { Brazil } \\
\text { Catanduva }\end{array}$ & $\begin{array}{l}\text { Every } 4^{\text {th }} \text { address of persons } 65+ \\
\text { residing in community selected (note: } \\
\text { nursing home residents screened but } \\
\text { not included); } 3 \text { stages (screening, } \\
\text { clinical assessment if scored low on } \\
\text { cognitive \& functional tests, \& } \\
\text { laboratory testing/ neuroimaging if } \\
\text { dementia suspected) }\end{array}$ & $\begin{array}{l}\text { All data obtained on subjects } \\
\text { evaluated by three } \\
\text { neurologists who determined } \\
\text { presence of dementia; } \\
\text { diagnosis of the specific } \\
\text { cause of dementia made on a } \\
\text { consensual basis by the } \\
\text { neurologists }\end{array}$ & $\begin{array}{l}\text { Dementia: DSM-IV }{ }^{40} \\
\text { DLB: McKeith }(1996)^{12}- \\
\text { not stated if possible } \\
\text { and/or probable }\end{array}$ & Not stated & $\begin{array}{l}1.2 \text { per } 1000 \text { ( } 2 \text { cases }) \\
\text { Comment: Additional } 4 \text { cases } \\
\text { of PDD; DLB accounted for } \\
1.7 \% \text { of dementia cases }\end{array}$ \\
\hline Ikeda $(2001)^{27}$ & $\begin{array}{l}65+(81.4 \\
\text { women/ } 82.3 \\
\text { men with } \\
\text { dementia) }\end{array}$ & $\begin{array}{l}\text { Western Pacific } \\
\text { Japan } \\
\text { Nakayama }\end{array}$ & $\begin{array}{l}\text { All persons } 65+\text { residing at home on } \\
\text { "prevalence day" (Jan. 1, 1997); } \\
3 \text { stages (screening, comprehensive } \\
\text { evaluation with medical record } \\
\text { review done if did poorly on any } \\
\text { cognitive screening measure or was } \\
\text { one of } 50 \text { who screened negative, \& } \\
\text { laboratory testing/ neuroimaging on } \\
\text { those who did poorly on neuropsy- } \\
\text { chology) }\end{array}$ & $\begin{array}{l}\text { Final diagnosis based on } \\
\text { information from } 3 \text { stages } \\
\text { (unclear who made and how } \\
\text { diagnosis made) }\end{array}$ & $\begin{array}{l}\text { Dementia: DSM-III-R }{ }^{39} \\
\text { DLB: McKeith }(1996)^{12} \\
\text { but not stated if possible } \\
\text { and/or probable }\end{array}$ & $1997-1998$ & $\begin{array}{l}0.9 \text { per } 1000 \text { ( } 1 \text { case }) \\
\text { Comment: DLB accounted for } \\
1.7 \% \text { of dementia cases }\end{array}$ \\
\hline
\end{tabular}




\begin{tabular}{|c|c|c|c|c|c|c|c|c|}
\hline 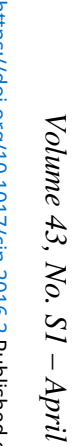 & Jhoo $(2008)^{29}$ & $65+(71.9)$ & $\begin{array}{l}\text { Western Pacific } \\
\text { Korea } \\
\text { Seongnam }\end{array}$ & $\begin{array}{l}\text { Random sample of roster of older } \\
\text { persons in community (response rate } \\
63.9 \% \text { ); all had comprehensive } \\
\text { evaluation including history, } \\
\text { examination, neuropsychology and } \\
\text { investigations; neuroimaging done if } \\
\text { dementia or stroke suspected done } \\
\text { on all }\end{array}$ & $\begin{array}{l}\text { Final diagnoses made by } 4 \\
\text { geropsychiatrists (unclear if } \\
\text { done individually or on } \\
\text { consensual basis) }\end{array}$ & $\begin{array}{l}\text { Dementia: DSM-IV }{ }^{40} \\
\text { DLB: McKeith }(1996)^{12} \\
\text { for possible and/or } \\
\text { probable }\end{array}$ & 2005-2006 & $\begin{array}{l}2.8 \text { per } 1000 \text { ( } 2 \text { cases) } \\
\text { Comment: Adjusted prevalence } \\
\text { for older population } \\
\text { estimated as } 4 \text { per } 1000 \text { ( } 95 \% \\
\text { CI 0-9); DLB accounted for } \\
5.4 \% \text { of dementia cases }\end{array}$ \\
\hline$\frac{\tilde{\sigma}}{a}$ & Spada $(2009)^{37}$ & $60-85(70.6)$ & $\begin{array}{l}\text { Europe } \\
\text { Italy } \\
\text { San Teodoro }\end{array}$ & $\begin{array}{l}\text { All persons } 60-85 \text { living in community } \\
\text { on list from municipal registry office } \\
\text { approached ( } 74.9 \% \text { participated); } \\
3 \text { stages (screening, clinical } \\
\text { assessment, and investigations if } \\
\text { required for suspected dementia } \\
\text { cases) }\end{array}$ & $\begin{array}{l}\text { Information on who made or } \\
\text { how diagnoses made not } \\
\text { provided }\end{array}$ & $\begin{array}{l}\text { Dementia: DSM-IV }{ }^{40} \\
\text { DLB: Not provided }\end{array}$ & 2005 & $\begin{array}{l}3.6 \text { per } 1000 \text { ( } 1 \text { case }) \\
\text { Comment: DLB accounted for } \\
5 \% \text { of dementia cases }\end{array}$ \\
\hline & Wada-Isoe $(2009)^{39}$ & $\begin{array}{c}65+(81.6 \\
\text { men/85 } \\
\text { women with } \\
\text { dementia })\end{array}$ & $\begin{array}{l}\text { Western Pacific } \\
\text { Japan } \\
\text { Ama-Cho }\end{array}$ & $\begin{array}{l}\text { Town residents } 65+; 2 \text { stages (screening } \\
\& \text { diagnostic evaluation of those who } \\
\text { screened positive) }\end{array}$ & $\begin{array}{l}\text { Based on clinical assessment, } \\
\text { laboratory investigations and } \\
\text { neuroimaging, but not clear } \\
\text { who made diagnosis }\end{array}$ & $\begin{array}{l}\text { Dementia: DSM-IV }{ }^{40} \\
\text { DLB: McKeith }(2005)^{14} \\
\text { but not stated if possible } \\
\text { and/or probable }\end{array}$ & 2008 & $\begin{array}{l}5.3 \text { per } 1000 \text { ( } 5 \text { cases) } \\
\text { Comment: Additional } 7 \text { cases } \\
\text { with PDD; DLB accounted } \\
\text { for } 4.8 \% \text { of dementia cases; } \\
\text { prevalence increased with } \\
\text { increasing age up to } 89\end{array}$ \\
\hline & Yamada $(2001)^{40}$ & $>65$ & $\begin{array}{l}\text { Western Pacific } \\
\text { Japan } \\
\text { Amino-cho }\end{array}$ & $\begin{array}{l}\text { Residents of community on prevalence } \\
\text { day (Jan. 1, 1998); } 2 \text { stages } \\
\text { (screening, comprehensive evaluation } \\
\text { (history, physical examination, } \\
\text { cognitive testing and laboratory/ } \\
\text { neuroimaging) on participants with } \\
\text { cognitive impairment) }\end{array}$ & $\begin{array}{l}\text { Information on who made and } \\
\text { how diagnoses made not } \\
\text { provided }\end{array}$ & $\begin{array}{l}\text { Dementia: DSM-III }{ }^{39} \\
\text { DLB: McKeith }(1996)^{12} \\
\text { for probable and } \\
\text { possible DLB }\end{array}$ & 1998 & $\begin{array}{l}1.1 \text { per } 1000 \text { ( } 4 \text { cases) } \\
\text { Comment: Additional } 3 \text { cases } \\
\text { of PDD; DLB accounted for } \\
2.8 \% \text { of dementia cases }\end{array}$ \\
\hline & \multicolumn{8}{|c|}{ Community and institution (listed alphabetically) } \\
\hline & $\begin{array}{c}\text { Gascón-Bayarri } \\
(2007)^{23}\end{array}$ & $70+(77.2)$ & $\begin{array}{l}\text { Europe } \\
\text { Spain } \\
\text { El Prat del } \\
\quad \text { Llobregat }\end{array}$ & $\begin{array}{l}\text { 43.2\% random age/ sex-stratified } \\
\text { sample of residents } 70+; 2 \text { stages } \\
\text { (screening \& diagnostic evaluation of } \\
\text { those who screened positive }+14.3 \% \\
\text { probability sample of those who } \\
\text { screened negative) }\end{array}$ & $\begin{array}{l}\text { Community: joint (neurologist } \\
\text { \& psychologist) diagnosis } \\
\text { based on clinical assessment } \\
\text { (where there was disagree- } \\
\text { ment 3rd neurologist made } \\
\text { diagnosis) } \\
\text { Institution: made by a physician } \\
\text { using clinical protocol }\end{array}$ & $\begin{array}{l}\text { Dementia: DSM-IV }{ }^{40} \\
\text { DLB: McKeith }(1996)^{12} \\
\text { but not stated if possible } \\
\text { and/or probable DLB }\end{array}$ & 2002-2003 & $\begin{array}{l}8.6 \text { per } 1000 \text { ( } 15 \text { cases) } \\
\text { Comment: There would be a } \\
\text { total of } 21 \text { cases if less } \\
\text { restrictive criteria used; } \\
\text { higher prevalence with } \\
\text { increasing age; DLB } \\
\text { accounted for } 9.1-12.7 \% \\
\text { (dependent on how } \\
\text { restrictive were the } \\
\text { diagnostic criteria) of } \\
\text { dementia cases }\end{array}$ \\
\hline & Harvey $(2003)^{25}$ & $<65(58.7)$ & $\begin{array}{l}\text { Europe } \\
\text { United Kingdom } \\
\text { London } \\
\quad \text { (4 boroughs) }\end{array}$ & $\begin{array}{l}\text { Multiple methods used to identify } \\
\text { potential cases (i.e., request for } \\
\text { notification of cases on a defined } \\
\text { "census" day by providers, search of } \\
\text { databases and case registries for } \\
\text { relevant ICD-9/10 diagnoses, hand } \\
\text { searching material provided by key } \\
\text { neurologists \& psychiatrists); } \\
\text { identified potential cases had their } \\
\text { medical records reviewed }\end{array}$ & $\begin{array}{l}\text { Based on collected information, } \\
\text { hierarchical diagnostic } \\
\text { algorithm used; in about half } \\
\text { of the dementia cases }(47 \%) \text {, } \\
\text { a detailed clinical assessment } \\
\text { was done to confirm } \\
\text { diagnosis; if diagnosis not } \\
\text { clear, discussion with } \\
\text { consensus by two of the } \\
\text { authors }\end{array}$ & $\begin{array}{l}\text { Dementia: DSM-IV }{ }^{40} \\
\text { DLB: McKeith }(1996)^{12} \\
\text { but not stated if possible } \\
\text { and/or probable but } \\
\text { authors' aim was to } \\
\text { make a "highest } \\
\text { confidence" single } \\
\text { diagnosis in each case }\end{array}$ & Not stated & $\begin{array}{l}0.06 \text { per } 1000 \text { persons } 30-64 \\
\text { (12 cases) } \\
\text { Comment: Additional } 2 \text { cases } \\
\text { of PDD; DLB accounted for } \\
7 \% \text { of cases }\end{array}$ \\
\hline
\end{tabular}


Table 1: (Continued)

\begin{tabular}{|c|c|c|c|c|c|c|c|}
\hline Author (date) & $\begin{array}{l}\text { Age range } \\
\text { (mean age) }\end{array}$ & $\begin{array}{l}\text { WHO geographic } \\
\text { region, country, \& } \\
\text { community }\end{array}$ & $\begin{array}{l}\text { Sample source and potential case } \\
\text { ascertainment process }\end{array}$ & $\begin{array}{l}\text { Method used to make } \\
\text { diagnosis }\end{array}$ & Diagnostic criteria used & $\begin{array}{l}\text { Date study } \\
\text { done }\end{array}$ & $\begin{array}{l}\text { Overall prevalence estimate } \\
\text { (\# cases) \& comments }\end{array}$ \\
\hline Ikejima $(2009)^{28}$ & 20-64 (56.9) & $\begin{array}{l}\text { Western Pacific } \\
\text { Japan } \\
\text { Ibaraki Prefecture }\end{array}$ & $\begin{array}{l}\text { Mailed questionnaire to a variety of } \\
\text { health organizations asking for } \\
\text { number of early-onset dementia cases } \\
\text { they care for; respondents of first } \\
\text { survey sent second questionnaire } \\
\text { requesting additional detailed } \\
\text { information }\end{array}$ & $\begin{array}{l}\text { Information provided by } \\
\text { healthcare organization } \\
\text { (as quality control exercise, } \\
9 \text { institutions with largest } \\
\text { number were identified \& } \\
\text { approximately half of the } \\
\text { cases reported were reviewed } \\
\text { internally by them to confirm } \\
\text { diagnosis) }\end{array}$ & $\begin{array}{l}\text { Dementia: DSM-III-R } \mathrm{R}^{30} \\
\text { DLB: McKeith (1996); }{ }^{12} \\
\text { not stated if possible } \\
\text { and/or probable; PDD } \\
\text { included as separate } \\
\text { group }\end{array}$ & 2006 & $\begin{array}{l}0.02 \text { per } 1000 \text { ( } 41 \text { cases of DLB } \\
\text { or PDD) } \\
\text { Comment: All cases occurred } \\
\text { in those older than } 50 \text { with } \\
\text { rising estimates with } \\
\text { increasing age up to } 64 \text {; } \\
\text { prevalence among those } 45- \\
64 \text { was } 0.05 \text { per } 1000 ; \text { DLB } \\
\text { or PDD accounted for } 6.2 \% \\
\text { of dementia cases }\end{array}$ \\
\hline Rahkonen $(2003)^{35}$ & $75-96(81.2)$ & $\begin{array}{l}\text { Europe } \\
\text { Finland } \\
\text { Kuopio }\end{array}$ & $\begin{array}{l}\text { Random sample of } 700 \text { residents born } \\
\text { before the first of Jan. } 1,1923 \text { and } \\
\text { living in community on Jan. 1, } 1998 \\
\text { (601 participated); structured } \\
\text { examination by geriatrician and } \\
\text { nurse, laboratory investigations, } \\
\text { review of medical records, and } \\
\text { interview of family/ health care staff } \\
\text { on participants }\end{array}$ & $\begin{array}{l}\text { Diagnosis confirmed at } \\
\text { consensus meetings using all } \\
\text { available data }\end{array}$ & $\begin{array}{l}\text { Dementia: DSM-IV }{ }^{40} \\
\text { DLB: McKeith }(1996)^{12} \\
\text { for probable and } \\
\text { possible DLB (PDD } \\
\text { included as separate } \\
\text { group with differential } \\
\text { from PDD made using } \\
\text { on "1-year" rule) }\end{array}$ & 1998 & $\begin{array}{l}33.3 \text { per } 1000 \text { ( } 20 \text { cases with } \\
\text { probable DLB) } \\
\text { Comment: } 30 \text { cases of probable } \\
\text { \& possible DLB; prevalence } \\
\text { based on cases of probable \& } \\
\text { possible DLB }=49.9 \text { per } \\
\text { 1000; } 2 \text { additional cases of } \\
\text { PDD; prevalence increased } \\
\text { with increasing age; probable } \\
\text { DLB accounted for } 14.6 \% \text { of } \\
\text { dementia cases (possible \& } \\
\text { probable DLB }=21.9 \% \text { ) }\end{array}$ \\
\hline Stevens $(2002)^{38}$ & $65+(75)$ & $\begin{array}{l}\text { Europe } \\
\text { United Kingdom } \\
\text { Islington }\end{array}$ & $\begin{array}{l}\text { Persons } 65+\text { identified by door-to-door } \\
\text { survey in randomly selected } \\
\text { enumeration districts; } 2 \text {-stage process } \\
\text { (screening \& diagnostic evaluation of } \\
\text { those who screened positive) }\end{array}$ & $\begin{array}{l}\text { Joint diagnosis by two raters } \\
\text { based on clinical assessment, } \\
\text { caregiver interview, \& } \\
\text { laboratory investigations }\end{array}$ & $\begin{array}{l}\text { Dementia: DSM-IV }{ }^{40} \& \\
\text { ICD-10 } 10^{42} \\
\text { DLB: McKeith }(1995)^{11} \\
\text { for probable and } \\
\text { possible DLB }\end{array}$ & Not stated & $\begin{array}{l}\text { 6.45 per } 1000 \text { ( } 7 \text { cases }) \\
\text { Comment: Additional } 15 \text { cases } \\
\text { were diagnosed with } \\
\text { possible DLB; probable } \\
\text { DLB accounted for } 10.9 \% \text { of } \\
\text { dementia cases ( } 34.4 \% \text { for } \\
\text { possible \& probable DLB) }\end{array}$ \\
\hline \multicolumn{8}{|c|}{ Institution only (assisted living, AL) } \\
\hline Rosenblatt $(2004)^{36}$ & $\begin{array}{l}\text { None (mean } \\
85.6)\end{array}$ & $\begin{array}{l}\text { Americas } \\
\text { United States } \\
\text { Maryland (city of } \\
\text { Baltimore and } \\
\text { several } \\
\text { Maryland } \\
\text { counties) }\end{array}$ & $\begin{array}{l}\text { Stratified (by size of facility), random } \\
\text { sample of both assisted living (AL) } \\
\text { facilities \& residents in central } \\
\text { Maryland (participation rates were } \\
67 \% \text { of facilities and } 74 \% \text { of residents } \\
\text { approached); detailed clinical } \\
\text { assessment and chart review } \\
\text { performed on all participating } \\
\text { residents }\end{array}$ & $\begin{array}{l}\text { Diagnosis made by consensus } \\
\text { panel after detailed review of } \\
\text { available information }\end{array}$ & $\begin{array}{l}\text { Dementia: DSM-IV } \\
\text { DLB: McKeith }(1992)^{10}\end{array}$ & Not stated & $\begin{array}{l}5 \text { per } 1000 \text { ( } 1 \text { case) } \\
\text { Comment: DLB accounted for } \\
0.7 \% \text { of dementia cases }\end{array}$ \\
\hline
\end{tabular}

*CDR = Clinical Dementia Rating.

$\dagger$ Diagnostic and Statistical Manual of Mental Disorders, 4th ed.

$\ddagger$ Parkinson’s disease dementia.

$\mathrm{DLB}=$ dementia with Lewy bodies. 
Table 2: Studies reporting on the incidence of dementia with Lewy bodies

\begin{tabular}{|c|c|c|c|c|c|c|c|}
\hline Author (date) & $\begin{array}{l}\text { Age range } \\
\text { (mean age) }\end{array}$ & $\begin{array}{l}\text { WHO geographic } \\
\text { region, country, } \\
\text { and community }\end{array}$ & $\begin{array}{l}\text { Sample source and potential case } \\
\text { ascertainment process }\end{array}$ & $\begin{array}{l}\text { Method used to make } \\
\text { diagnosis }\end{array}$ & Diagnostic criteria used & $\begin{array}{l}\text { Date study } \\
\text { done }\end{array}$ & $\begin{array}{l}\text { Overall incidence rate (\# cases) \& } \\
\text { comments }\end{array}$ \\
\hline \multicolumn{8}{|c|}{ Incidence rate (listed alphabetically) } \\
\hline Matsui $(2009)^{30}$ & $65+$ & $\begin{array}{l}\text { Western Pacific } \\
\text { Japan } \\
\text { Hisayama }\end{array}$ & $\begin{array}{l}\text { All residents in town } 65+\text {; detected by } \\
\text { continuous monitoring \& annual } \\
\text { assessments of residents not } \\
\text { demented at baseline }\end{array}$ & $\begin{array}{l}\text { Based on clinical assessment } \\
\text { and review of medical } \\
\text { records but not clear who } \\
\text { made the diagnosis; } \\
\text { autopsies conducted on } 60 \% \\
\text { of dementia cases }\end{array}$ & $\begin{array}{l}\text { Dementia: DSM-III-R }{ }^{39} \\
\text { DLB: McKeith }(2005)^{14} \\
\text { for possible, probable \& } \\
\text { definite DLB }\end{array}$ & $1985-2002$ & $\begin{array}{l}1.4 \text { per } 1000 \text { person years ( } 12 \text { cases) } \\
\text { Comment: Additional } 17 \text { cases of } \\
\text { DLB with AD and/or other central } \\
\text { nervous system conditions; DLB } \\
\text { accounted for } 4.4 \% \text { of all dementia } \\
\text { cases; no increase in incidence seen } \\
\text { with increasing age; trend to higher } \\
\text { mortality with DLB }\end{array}$ \\
\hline Meguro $(2007)^{31}$ & $65+$ & $\begin{array}{l}\text { Western Pacific } \\
\text { Japan } \\
\text { Tajiri }\end{array}$ & $\begin{array}{l}\text { Non-demented (at baseline) older } \\
\text { residents enrolled in a study were } \\
\text { reassessed at either } 5 \text { years if MRI } \\
\text { done or } 7 \text { years if did not have MRI } \\
\text { (data on specific type limited to } \\
5 \text {-year group); demographic, lifestyle, } \\
\text { social support and medical data were } \\
\text { collected on participants, as well as a } \\
\text { physical examination; Clinical } \\
\text { Dementia Rating (CDR) and } \\
\text { cognitive testing were done }\end{array}$ & $\begin{array}{l}\text { Dementia diagnosis made by } \\
\text { CDR score of } 1+; \text { not clear } \\
\text { who made or how specific } \\
\text { diagnosis of dementia cause } \\
\text { made, which was limited to } \\
\text { those who had an MRI done }\end{array}$ & $\begin{array}{l}\text { Dementia: DSM-IV } \\
\text { DLB: McKeith }(2005)^{14} \\
\text { but not stated if possible } \\
\text { and/or probable }\end{array}$ & $1998-2003$ & $\begin{array}{l}1.6 \text { per } 1000 \text { person years ( } 2 \text { cases) } \\
\text { Comment: DLB accounted for } 7.1 \% \\
\text { of all dementia cases }\end{array}$ \\
\hline Miech $(2002)^{32}$ & $\begin{array}{l}65+(74 \text { on } \\
\text { entry) }\end{array}$ & $\begin{array}{l}\text { Americas } \\
\text { United States } \\
\text { Cache County, } \\
\text { Utah }\end{array}$ & $\begin{array}{l}\text { Non-demented (at baseline) community } \\
\text { residents } 65+(\text { Jan. } 1 / 95 \text { ) enrolled in } \\
\text { study reassessed in } 3 \text { years; } 3 \text {-stage } \\
\text { process (screening, detailed clinical } \\
\text { assessment if dementia suspected }+ \\
\text { stratified probability subsample, and } \\
\text { investigations including } \\
\text { neuroimaging if suspected dementia } \\
\text { or substantive cognitive difficulty) }\end{array}$ & $\begin{array}{l}\text { Final clinical diagnoses made at } \\
\text { consensus conferences that } \\
\text { included geropsychiatrists, } \\
\text { neurologists, neuropsycholo- } \\
\text { gists, and neuroscientists; } \\
\text { final clinical diagnoses could } \\
\text { be revised based on autopsy } \\
\text { findings }\end{array}$ & $\begin{array}{l}\text { Dementia: modified DSM- } \\
\text { III-R }{ }^{39} \text { (did not require } \\
\text { deficit in both short- and } \\
\text { long-term memory) } \\
\text { DLB: criteria not given }\end{array}$ & $1995-1999$ & $\begin{array}{l}0.6 \text { per } 1000 \text { person years (six definite } \\
\text { or possible DLB cases alone or in } \\
\text { combination with other dementing } \\
\text { illness) } \\
\text { Comment: Additional } 2 \text { cases of } \\
\text { possible AD/Parkinson's, } 1 \text { case of } \\
\text { possible vascular dementia/ } \\
\text { Parkinson's, and } 9 \text { of PDD; DLB } \\
\text { accounted for } 3.2 \% \text { of all dementia } \\
\text { cases }\end{array}$ \\
\hline Nitrini $(2004)^{33}$ & $65+$ & $\begin{array}{l}\text { Americas } \\
\text { Brazil } \\
\text { Catanduva }\end{array}$ & $\begin{array}{l}\text { Non-demented (at baseline) community } \\
\text { residents enrolled in study (see } \\
\text { Herrera }^{26} \text { ) reassessed } 3.25 \text { years after } \\
\text { entry; } 3 \text { stages (screening, clinical } \\
\text { assessment if score low on cognitive } \\
\& \text { functional tests + random sample } \\
\text { of those who screened negative, and } \\
\text { laboratory/neuroimaging if suspected } \\
\text { dementia) }\end{array}$ & $\begin{array}{l}\text { Diagnosis made by consensus } \\
\text { of entire clinical team }\end{array}$ & $\begin{array}{l}\text { Dementia: DSM-IV }{ }^{40} \\
\text { DLB: McKeith }(1996)^{12} \\
\text { but not stated if possible } \\
\text { and/or probable }\end{array}$ & $1997-2000$ & $\begin{array}{l}0.5 \text { per } 1000 \text { person years ( } 2 \text { cases) } \\
\text { Comment: DLB accounted for } 4 \% \text { of } \\
\text { all dementia cases; additional } \\
3 \text { cases with PDD }\end{array}$ \\
\hline Perez $(2010)^{34}$ & $\begin{array}{l}65+(75.5 \text { on } \\
\text { entry })\end{array}$ & $\begin{array}{l}\text { Europe } \\
\text { France } \\
\text { Gironde and } \\
\text { Dordogne }\end{array}$ & $\begin{array}{l}\text { Random sample of residents } 65+\text {; } \\
\text { 2-stage process (screening \& } \\
\text { diagnostic evaluation if screened } \\
\text { positive) }\end{array}$ & $\begin{array}{l}\text { Consensus diagnosis made after } \\
\text { review of all available } \\
\text { clinical data }\end{array}$ & $\begin{array}{l}\text { Dementia: DSM-III-R } \\
\text { DLB: if both dementia and } \\
\text { parkinsonism present; } \\
\text { McKeith }(2005)^{14} \\
\text { criteria for possible \& } \\
\text { probable DLB used }\end{array}$ & $1988-2003$ & $\begin{array}{l}0.5 \text { for probable DLB and } 1.1 \text { per } \\
1000 \text { person years for probable \& } \\
\text { possible DLB ( } 13 \text { cases probable } \\
\text { DLB \& } 29 \text { probable/possible DLB) } \\
\text { Comment: Additional } 9 \text { cases of } \\
\text { AD/parkinsonism; probable \& } \\
\text { possible DLB accounted for } 4.5 \% \\
\text { of all dementia cases and rising } \\
\text { incidence with increasing age }\end{array}$ \\
\hline
\end{tabular}


Table 3: Quality assessment scores of dementia with Lewy Bodies incidence and prevalence studies included in the systematic review

\begin{tabular}{|c|c|c|c|c|c|c|c|c|c|}
\hline Study (year) & $\begin{array}{l}\text { Q1: Target } \\
\text { population } \\
\text { described? }\end{array}$ & $\begin{array}{c}\text { Q2: Cases from } \\
\text { entire } \\
\text { population or } \\
\text { probability } \\
\text { sampling? } \\
\end{array}$ & $\begin{array}{l}\text { Q3: Response } \\
\text { rate }>70 \% \text { ? }\end{array}$ & $\begin{array}{l}\text { Q4: Non- } \\
\text { responders } \\
\text { clearly } \\
\text { described? }\end{array}$ & $\begin{array}{l}\text { Q5: Sample } \\
\text { representative } \\
\text { of population? }\end{array}$ & $\begin{array}{c}\text { Q6: Data } \\
\text { collection } \\
\text { methods } \\
\text { standardized? }\end{array}$ & $\begin{array}{l}\text { Q7: Validated } \\
\text { criteria to } \\
\text { assess disease? }\end{array}$ & $\begin{array}{c}\text { Q8: Were } \\
\text { estimates given } \\
\text { with confidence } \\
\text { intervals or } \\
\text { subgroups? } \\
\end{array}$ & $\begin{array}{c}\text { Total quality } \\
\text { score (out of 8) }\end{array}$ \\
\hline Bennett $(2003)^{19}$ & No & Yes & NR & No & NR & Yes & Yes & No & 4 \\
\hline Bottino $(2008)^{20}$ & Yes & No & No & No & $\mathrm{NC}$ & Yes & Yes & Yes & 4 \\
\hline de Jesus Llibre $(2009)^{21}$ & Yes & Yes & Yes & No & NR & Yes & Yes & Yes & 6 \\
\hline de Silva $(2003)^{22}$ & Yes & Yes & NR & NR & NR & Yes & Yes & Yes & 5 \\
\hline Gascon-Bayarri $(2007)^{23}$ & Yes & Yes & Yes & Yes & Yes & Yes & Yes & Yes & 8 \\
\hline Gurvit $(2008)^{24}$ & Yes & Yes & Yes & Yes & Yes & Yes & Yes & Yes & 8 \\
\hline Harvey $(2003)^{25}$ & Yes & Yes & NA & No & $\mathrm{NC}$ & Yes & Yes & Yes & 5 \\
\hline Herrera $(2002)^{26}$ & Yes & Yes & Yes & No & $\mathrm{NC}$ & Yes & Yes & Yes & 6 \\
\hline Ikeda $(2001)^{27}$ & Yes & Yes & Yes & Yes & Yes & Yes & Yes & No & 7 \\
\hline Ikejima $(2009)^{28}$ & Yes & Yes & NR & NR & $\mathrm{NC}$ & Yes & Yes & Yes & 5 \\
\hline Jhoo $(2008)^{29}$ & Yes & Yes & No & Yes & No & Yes & Yes & Yes & 6 \\
\hline Matsui $(2009)^{30}$ & Yes & Yes & Yes & No & $\mathrm{NC}$ & Yes & Yes & No & 5 \\
\hline Meguro (2007) ${ }^{31}$ & Yes & Yes & No & Yes & No & Yes & Yes & Yes & 6 \\
\hline $\operatorname{Miech}(2002)^{32}$ & Yes & Yes & Yes & Yes & Yes & Yes & Yes & Yes & 8 \\
\hline Nitrini $(2004)^{33}$ & Yes & Yes & NR & Yes & Yes & Yes & Yes & Yes & 7 \\
\hline Perez $(2010)^{34}$ & Yes & Yes & No & No & NR & Yes & Yes & Yes & 5 \\
\hline Rahkonen $(2003)^{35}$ & Yes & Yes & Yes & Yes & No & Yes & Yes & Yes & 7 \\
\hline Rosenblatt $(2004)^{36}$ & Yes & Yes & Yes & No & $\mathrm{NR}$ & Yes & Yes & No & 5 \\
\hline Spada $(2009)^{37}$ & Yes & Yes & Yes & No & NR & Yes & Yes & No & 5 \\
\hline Stevens $(2002)^{38}$ & Yes & Yes & Yes & Yes & NR & Yes & Yes & Yes & 7 \\
\hline Wada-Isoe $(2009)^{39}$ & Yes & Yes & NR & No & Yes & Yes & Yes & Yes & 6 \\
\hline Yamada $(2001)^{40}$ & No & Yes & NR & No & NR & Yes & Yes & No & 4 \\
\hline
\end{tabular}

$\mathrm{NR}=$ not reported; $\mathrm{NC}=$ not clear 
reported on study populations $<65$ years of age. ${ }^{25,28}$ Of those dealing with older populations, the lower age thresholds were $60+,{ }^{20,37} 65+,{ }^{21,22,26,27,29-34,38-40} 70+,{ }^{23,24} 75+{ }^{35}$ and $81+{ }^{19}$ The final study did not employ an age range but dealt with an institutional population (mean age 85.6 years). ${ }^{36}$

Fourteen studies used a multistage approach (i.e., initial screening assessment followed by an in-depth evaluation of those who screened positive for dementia \pm a random sample of those screening negative) to detect cases. Most $(n=20)$ studies used either DSM-III-R ${ }^{41}$ or DSM-IV ${ }^{42}$ criteria for the diagnosis of dementia. The two exceptions were one ${ }^{19}$ that utilized Clinical Dementia Rating $(\mathrm{CDR})^{43}$ scores and another ${ }^{38}$ that used both DSM-IV and ICD-10 criteria. $^{44}$ The disciplinary backgrounds of those performing clinical assessments were diverse and included (listed alphabetically) geriatric psychiatry, geriatrics, internal medicine, neurology (including residents in neurology), neuropsychology, neuropsychiatry, nursing, physiatry/disability medicine, psychiatry, physician (no specialty specified), psychology and psychometry. A consensus approach (i.e., more than one person involved) was used to make final diagnoses in 11 studies. $^{20,21,23,24,26,32-36,38}$

Where stated (three studies did not provide information), ${ }^{20,32,37}$ various versions of criteria developed by McKeith et al. ${ }^{10-14}$ were used for diagnosing DLB. Fourteen studies did not indicate whether probable or both possible and probable DLB cases were being enumerated. $^{20,21,23-28,31-33,36,37,39}$ An incidence study $^{30}$ and two prevalence studies ${ }^{29,40}$ reported on the number of possible and probable DLB cases, while an incidence study ${ }^{34}$ and four prevalence ones $^{19,22,35,38}$ gave the number of probable DLB cases (four of these studies also provided data on possible DLB cases). The specific methods used to identify hallucinations were provided by ten studies ${ }^{19,23,25,27,29,32-36}$ (in seven, this was by the administration of a standardized behavioural instrument such as the Neuropsychiatric Inventory ${ }^{45}$ ). Four studies gave information on detection of

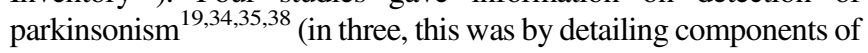
the clinical assessment), and three provided the approach used for fluctuations (principally by asking subjects and informants). ${ }^{19,34,35}$ While four studies ${ }^{30,31,34,39}$ used the latest revision of the consensus criteria $^{16}$ that included suggestive features, no study described the methods used for their detection.

Seventeen studies did not address possible overlap between Alzheimer's disease (AD) and DLB. The five that did used dissimilar methodologies and reported on differing aspects of that relationship. Though the total number of cases per study was small, it was reported that a third ${ }^{23}$ to a half ${ }^{38}$ of DLB cases also met AD criteria, that the ratio of "combined" AD and DLB to "pure" DLB ranged from $0.75^{30}$ to $7.0,^{32}$ and that the ratio of AD with parkinsonism to probable DLB was $0.69 .^{34}$

Eleven studies gave data on Parkinson's disease dementia (PDD) as well as DLB, ${ }^{20,21,25,26,28,32,33,35,38-40}$ with seven providing the criteria used to diagnose PDD (i.e., $\operatorname{six}^{20,21,25,28,35,38}$ utilized DSM-IV criteria ${ }^{42}$ and another ${ }^{39}$ used published PDD criteria $\left.^{46}\right)$. In one report, ${ }^{35}$ the authors adhered to the "one-year rule" in distinguishing DLB from PDD,${ }^{14,16}$ while in another, ${ }^{38}$ no restriction on the duration of parkinsonism prior to the onset of dementia was used (the remaining studies did not comment on this issue). In three studies, ${ }^{35,38,40}$ DLB was more common than PDD, while PDD was more common in five $\mathrm{e}^{21,26,32,39}$ (the conditions in two studies ${ }^{20,25}$ were equally common, and the final one ${ }^{28}$ made no attempt to distinguish between the two).
Among those aged $65+$ residing in the community, DLB incidence rates ranged from 0.5 to 1.6 per 1000 person-years. Little could be said about the influence of age on incidence rates, as all incidence studies dealt with similarly aged individuals (i.e., 65+). One study reported that no increase in incidence was observed with increasing age. ${ }^{30}$ All incidence studies scored 5+ on the quality assessment.

DLB accounted for 3.2 to $7.1 \%$ of incident dementia cases (mean 4.6\%, SD 1.5). The percentages ascribed to DLB were similar in the study ${ }^{31}$ that used 1996 consensus criteria ${ }^{12}$ compared to the three ${ }^{28,29,32}$ that utilized the 2005 revision $^{14}$ (4 and $5.3 \%$, respectively). Likewise, the percentages were similar when the two studies published in 2005 or earlier (3.6\%) were compared to the three published in 2006 or later $(5.3 \%)$. The one study that reported on both probable and possible cases ${ }^{34}$ attributed $4.5 \%$ of dementia cases to DLB if both were included, compared to $2.0 \%$ if cases were restricted to those with probable DLB.

Point and period prevalence estimates varied more widely: from a low of 0.02 in a community and institutional study from Japan to a high of 63.5 per 1000 in a community study from Australia (Figures 2 and 3). If analysis was limited to the 12 studies scoring $5+$ on the quality assessment, prevalence per 1000 varied from 0.02 to 33.3 per 1000 (Table 3). ${ }^{20-24,28,29,35-39}$ Estimates rose with increasing age. In studies restricted to participants less than 65 years of age, prevalence was $0.02-0.06$ per 1000 compared to 0.3-6.45 when participants were 60 or $65+$, 8.6-33.3 among those 70+, and 63.5 in the one study limited to those $81+$. This latter study ${ }^{19}$ had the highest reported prevalence of DLB and a high proportion of dementia cases attributed to this condition (probable and possible DLB accounted for $53 / 78$ [67.9\%] of all dementia cases). Other unique features of the study included the use of CDR criteria to detect dementia and the severity of dementia among participants diagnosed with DLB (mean Mini-Mental State Examination [MMSE] score of 12.6). This study was assigned a quality score of 4 (see Table 3 ) because of methodological concerns (i.e., a single non-examining physician made the diagnosis based on a review of assessment information). ${ }^{19}$ The study with the second highest prevalence reported on the second oldest group of participants, ${ }^{35}$ and, again, when present, dementia was of moderate severity (mean MMSE score of 14.1).

DLB made up between 0.3 and $24.4 \%$ of all cases of dementia in the prevalence studies when the most restrictive definition for DLB provided was used (mean $6.4 \%, S D$ 6.1). The proportions were similar in the 11 studies $^{19,22-29,35,40}$ using 1996 consensus criteria $^{12}$ and the one ${ }^{39}$ that utilized the 2005 revision ${ }^{14}$ (7.8 and $5.3 \%$, respectively). Likewise, the percentages were similar when the nine studies published in 2005 or earlier $(7.5 \%)$ were compared with the eight reports published in 2006 or later $(4.4 \%)$. Three studies provided information on both probable and possible DLB cases. ${ }^{19,35,38}$ Compared to restricting case definition to only probable DLB, including possible and probable cases increased by 1.5-3.2 fold the proportion of dementia cases attributed to DLB.

\section{Discussion}

We updated and/or expanded on prior systematic reviews on the incidence and prevalence of DLB. ${ }^{15,16}$ This allowed us to highlight a number of methodological issues that need to be 


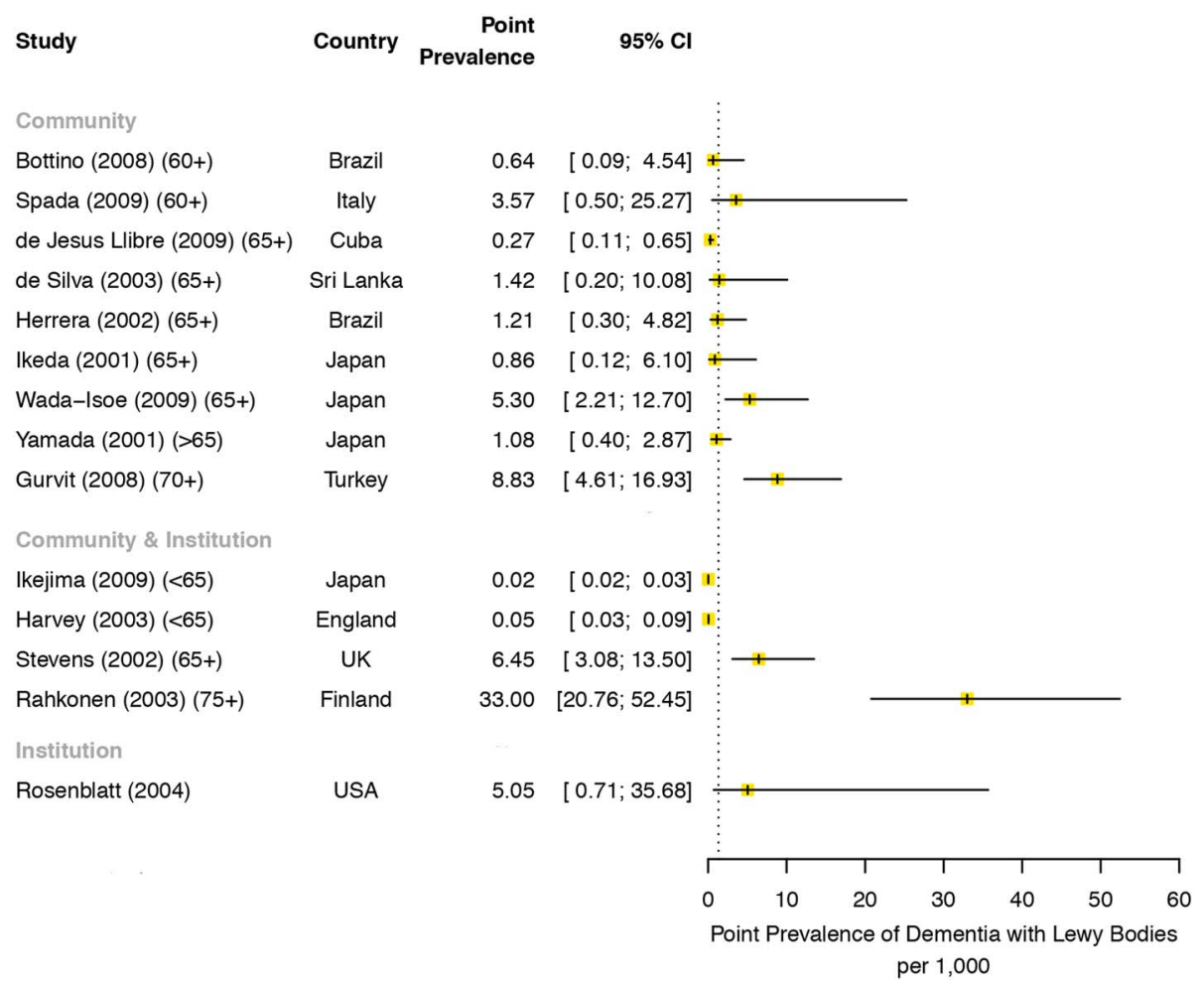

Figure 2: Point prevalence of dementia with Lewy bodies (listed in order from the youngest to the oldest age range for the study population).

addressed in order to derive more precise estimates of the true burden of this form of dementia. We could not perform a pooled meta-analysis due to the significant between-study heterogeneity and small sample size. While we found that DLB incidence rates ranged from 0.5 to 1.6 per 1000 person-years, prevalence varied more widely and ranged from 0.02 to 33.3 per 1000 even when restricted to higher-quality studies. The two other DLB systematic reviews ${ }^{15,16}$ reported similar results, while one that dealt with PDD estimated that in the general population in the 65+ group its prevalence was between 2 and 5 per $1000 .^{47}$

The uncertainty about the prevalence of DLB is at least partially due to methodological issues. Most of the included studies $(n=18)$ were not designed to specifically investigate the incidence and/or prevalence of DLB. The cognitive testing used to screen for cases had been primarily selected to detect AD (see Tables 1 and 2). How well they worked in detecting potential cases of DLB is unknown. The differing diagnostic criteria for dementia used would influence how often dementia would be detected. ${ }^{48}$ While a clinic-based study reported that the 2005 consensus criteria for DLB identified more individuals with a dementia as suffering from probable DLB compared to the 1996 version (essentially by classifying more cases as probable and fewer as possible DLB), ${ }^{3}$ it is unknown whether they are more accurate. There is a suggestion that the diagnostic error rate for DLB has recently increased. ${ }^{49} \mathrm{We}$ did not find marked differences between the two versions of diagnostic criteria in terms of the

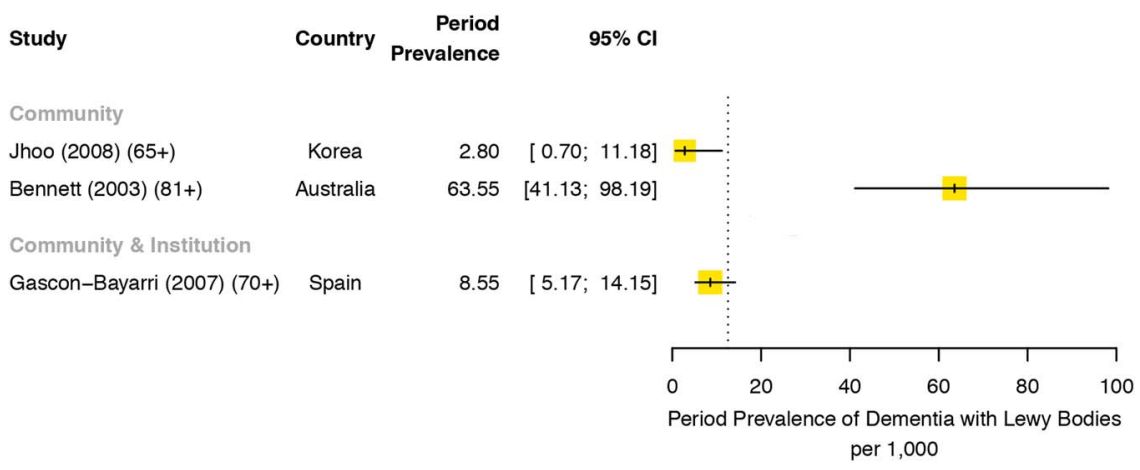

Figure 3: Period prevalence of dementia with Lewy bodies (listed in order from the youngest to the oldest age range for the study population). 
proportion of those with dementia identified as having DLB. How diagnostic criteria are operationalized is likely of more importance than which specific one is employed. Most of the studies we examined $(n=14)$ did not specify whether they were reporting on probable DLB or possible as well, and they provided little if any information on how core and suggestive features of DLB were detected. Challenges in assessing these features (especially fluctuations) contribute to the difficulties encountered in accurately diagnosing DLB. ${ }^{13}$ The age ranges studied varied markedly. With an age-associated condition like DLB, this would have an effect on the estimates provided, which is what we found. Finally, the number of cases upon which estimates were based was often small (see Tables 1 and 2), thus decreasing their precision. ${ }^{50}$

Issues exist with the consensus clinical criteria used for diagnosis, as the core and suggestive features are not specific to DLB. For example, parkinsonism, especially if mild, can be found with normal aging, idiopathic Parkinson's disease and AD. ${ }^{51}$ Autopsy-based validation studies of these diagnostic criteria have shown variable but often low sensitivity and high specificity with stage-dependent false negative (usually early in the course of the illness) and false positive errors (especially with advanced dementia). ${ }^{49}$

Particular challenges arise when there are coexisting pathologies such as AD. ${ }^{52,53}$ The complex relationship between DLB and AD is still being elucidated. Patients with DLB commonly have $\beta$-amyloid deposition and diffuse plaque formation without a significant burden of neocortical neurofibrillary tangles. ${ }^{1,14}$ Concomitant Alzheimer's pathology is not excluded by a diagnosis of either probable or possible DLB. Another issue is the relationship between PDD and DLB. They are typically differentiated by the arbitrary "one-year rule" (i.e., DLB is diagnosed if dementia occurs within 12 months of the onset of extrapyramidal motor symptoms, while the diagnosis is PDD if the clinical history of parkinsonism is longer than a year). ${ }^{12,14}$ The clinical and pathological overlaps with both Alzheimer's and Parkinson's raises uncertainty in the diagnosis of DLB. The term DLB, while acknowledging the presence of Lewy bodies, does not specify their relative importance compared to other brain pathologies in explaining a patient's cognitive symptoms. It is not clear how cases with presumed "mixed" aetiologies, which are common with Lewy body disease, should be dealt with. As a minimum, the approach taken by the investigators should be described. This was often not mentioned in the studies reviewed. Validated biomarkers for DLB could improve on the accuracy of diagnosing DLB but are not generally available for clinical use. ${ }^{54}$ Biomarkers may not be practical for population-based studies, and difficulties would remain in dealing with cases having more than one contributing aetiology and distinguishing DLB from PDD and atypical parkinsonian syndromes.

Notwithstanding these limitations, we can conclude that clinically defined DLB becomes more common with increasing age and accounts for about $5 \%$ of all dementia cases encountered in older populations. This updated information can be used in the planning of healthcare services and educational programs for practitioners. DLB presents unique diagnostic challenges that likely contributed to the wide variation in reported rates found in the literature reviewed. Standardization in the collection and reporting of data, how diagnostic criteria are operationalized and the approach taken in dealing with the borders between DLB and both AD and PDD would improve on the reliability and comparability of future epidemiologic studies of this condition.

\section{ACKNOWLEDGEMENTS}

We are thankful to Ms. Diane Lorenzetti, librarian at the University of Calgary, who guided the development of the search strategy for this systematic review. Our study is part of the National Population Health Study of Neurological Conditions. We wish to acknowledge the membership of Neurological Health Charities Canada and the Public Health Agency of Canada for their contribution to the success of this initiative. Funding for the study was provided by the Public Health Agency of Canada. The opinions expressed in this publication are those of the authors/researchers and do not necessarily reflect the official views of the Public Health Agency of Canada.

\section{Disclosures}

Nathalie Jetté has the following disclosures: Public Health Agency of Canada, Principal Investigator, research support; Canada Research Chair, Researcher, research support; Alberta Innovates Health Solutions, Researcher, research support.

David Hogan holds the Brenda Strafford Foundation Chair in Geriatric Medicine, though receives no salary support from this.

Kirsten Fiest, Jodie Roberts, Colleen Maxwell, Jonathan Dykeman, Tamara Pringsheim, Thomas Steeves, Eric Smith and Dawn Pearson do not have anything to disclose.

\section{Statement of Authorship}

DBH, KMF, JIR, CJM, TP and NJ contributed to study conception and design. DBH, KMF, JIR, CJM, TS, EES, DP and NJ contributed to acquisition of the data. DBH, KMF and JD conducted the data analysis. DBH, KMF, JIR, CJM, EES and NJ participated in interpretation of the study data. All authors participated in critically revising the manuscript for important intellectual content and gave final approval for the submission of this manuscript and any further submissions of this work.

\section{SUPPlementary Material}

To view supplementary material for this article, please visit http://dx.doi.org/doi:10.1017/cjn.2016.2

\section{REFERENCES}

1. McKeith I, Mintzer J, Aarsland D, Burn D, Chiu H, Cohen-Mansfield J, et al. Dementia with Lewy bodies. Lancet Neurol. 2004;3(1): 19-28.

2. Feldman H, Levy AR, Hsiung GY, Peters KR, Donald A, Black SE, et al. A Canadian cohort study of cognitive impairment and related dementias (ACCORD): study methods and baseline results. Neuroepidemiology. 2003;22(5):265-74.

3. Aarsland D, Rongve A, Nore SP, Skogseth R, Skulstad S, Ehrt U, et al. Frequency and case identification of dementia with Lewy bodies using the revised consensus criteria. Dement Geriatr Cogn Disord. 2008;26(5):445-52.

4. Woodward MC, Woodward E. A national survey of memory clinics in Australia. Int Psychogeriatr. 2009;21(4):696-702.

5. Alladi S, Mekala S, Chadalawada SK, Jala S, Mridula R, Kaul S. Subtypes of dementia: a study from a memory clinic in India. Dement Geriatr Cogn Disord. 2011;32(1):32-8.

6. Kokmen E, Ozsarfati Y, Beard CM, O'Brien PC, Rocca WA. Impact of referral bias on clinical and epidemiological studies of Alzheimer's disease. J Clin Epidemiol. 1996;49(1): 79-83. 
7. Sonnen JA, Larson EB, Crane PK, Haneuse S, Li G, Schellenberg GD, et al. Pathological correlates of dementia in a longitudinal, population-based sample of aging. Ann Neurol. 2007;62(4): 406-13.

8. Matthews FE, Brayne C, Lowe J, McKeith I, Wharton SB, Ince P. Epidemiological pathology of dementia: attributable risks at death in the Medical Research Council Cognitive Function and Ageing Study. PLoS Med. 2009;6(11):e1000180.

9. Byrne EJ, Lennox G, Godwin-Austen RB, Jefferson D, Lowe J, Mayer RJ, et al. Dementia associated with cortical Lewy bodies: proposed clinical diagnostic criteria. Dementia. 1991;2:283-4.

10. McKeith IG, Perry RH, Fairbairn AF, Jabeen S, Perry EK. Operational criteria for senile dementia of Lewy body type (SDLT). Psychol Med. 1992;22(4):911-22.

11. McKeith IG, Galasko D, Wilcock GK, Byrne EJ. Lewy body dementia: diagnosis and treatment. Br J Psychiatry. 1995;167(6): 709-17.

12. McKeith IG, Galasko D, Kosaka K, Perry EK, Dickson DW, Hansen LA, et al. Consensus guidelines for the clinical and pathologic diagnosis of dementia with Lewy bodies (DLB): report of the consortium on DLB international workshop. Neurology. 1996;47(5):1113-24

13. McKeith IG, Perry EK, Perry RH. Report of the second Dementia with Lewy Body International Workshop: diagnosis and treatment. Consortium on Dementia with Lewy Bodies. Neurology. 1999;53(5):902-5.

14. McKeith IG, Dickson DW, Lowe J, Emre M, O’Brien JT, Feldman H, et al. Diagnosis and management of dementia with Lewy bodies: third report of the DLB Consortium. Neurology. 2005;65(12): 1863-72.

15. Zaccai J, McCracken C, Brayne C. A systematic review of prevalence and incidence studies of dementia with Lewy bodies. Age Ageing. 2005;34(6):561-6.

16. Vann Jones SA, O'Brien JT. The prevalence and incidence of dementia with Lewy bodies: a systematic review of population and clinical studies. Psychol Med. 2013:1-11.

17. Boyle MH. Guidelines for evaluating prevalence studies. Evid Based Mental Health. 1998;1:37-9.

18. Loney PL, Chambers LW, Bennett KJ, Roberts JG, Stratford PW. Critical appraisal of the health research literature: prevalence or incidence of a health problem. Chronic Dis Can. 1998;19(4): 170-6.

19. Bennett HP, Piguet O, Grayson DA, Creasey H, Waite LM, Broe GA, et al. A 6-year study of cognition and spatial function in the demented and non-demented elderly: the Sydney Older Persons Study. Dement Geriatr Cogn Disord. 2003;16:181-6.

20. Bottino CMC, Azevedo D Jr, Tatsch M, Hototian SR, Moscoso MA, Folquitto J, et al. Estimate of dementia prevalence in a community sample from São Paulo, Brazil. Dement Geriatr Cogn Disord. 2008;26(4):291-9.

21. de Jesus Llibre J, Fernandez Y, Marcheco B, Contreras N, Lopez AM, Otero M, et al. Prevalence of dementia and Alzheimer's disease in a Havana municipality: a community-based study among elderly residents. MEDICC Rev. 2009;11(2):29-35.

22. de Silva HA, Gunatilake SB, Smith AD. Prevalence of dementia in a semi-urban population in Sri Lanka: report from a regional survey. Int J Geriatr Psychiatry. 2003;18(8):711-5.

23. Gascon-Bayarri J, Rene R, Del Barrio JL, De Pedro-Cuesta J, Ramon JM, Manubens JM, et al. Prevalence of dementia subtypes in El Prat de Llobregat, Catalonia, Spain: the PRATICON study. Neuroepidemiology. 2007;28(4):224-34

24. Gurvit H, Emre M, Tinaz S, Bilgic B, Hanagasi H, Sahin H, et al. The prevalence of dementia in an urban Turkish population. Am J Alzheimers Dis Other Demen. 2008;23(1):67-76.

25. Harvey RJ, Skelton-Robinson M, Rossor MN. The prevalence and causes of dementia in people under the age of 65 years. J Neurol Neurosurg Psychiatry. 2003;74(9):1206-9.

26. Herrera E Jr, Caramelli P, Silveira ASB, Nitrini R. Epidemiologic survey of dementia in a community-dwelling Brazilian population. Alzheimer Dis Assoc Disord. 2002:16(2):103-8.

27. Ikeda M, Hokoishi K, Maki N, Nebu A, Tachibana N, Komori K, et al. Increased prevalence of vascular dementia in Japan: a community-based epidemiological study. Neurology. 2001;57(5): $839-44$.

28. Ikejima C, Yasuno F, Mizukami K, Sasaki M, Tanimukai S, Asada T. Prevalence and causes of early-onset dementia in Japan: a population-based study. Stroke. 2009;40(8):2709-14.

29. Jhoo JH, Kim KW, Huh Y, Lee SB, Park JH, Lee JJ, et al. Prevalence of dementia and its subtypes in an elderly urban Korean population: results from the Korean Longitudinal Study on Health And Aging (KLoSHA). Dement Geriatr Cogn Disord. 2008; 26(3):270-6.

30. Matsui Y, Tanizaki Y, Arima H, Yonemoto K, Doi Y, Ninomiya T, et al. Incidence and survival of dementia in a general population of Japanese elderly: the Hisayama study. J Neurol Neurosurg Psychiatry. 2009;80(4):366-70.

31. Meguro $K$, Ishii $H$, Kasuya $M$, Akanuma $K$, Meguro $M$, Kasai M, et al. Incidence of dementia and associated risk factors in Japan: the Osaki-Tajiri Project. J Neurol Sci. 2007;260(1-2): $175-82$.

32. Miech RA, Breitner JCS, Zandi PP, Khachaturian AS, Anthony JC, Mayer L. Incidence of AD may decline in the early 90 s for men, later for women: the Cache County study. Neurology. 2002; 58(2):209-18

33. Nitrini R, Caramelli P, Herrera E Jr, Bahia VS, Caixeta LF, Radanovic $\mathrm{M}$, et al. Incidence of dementia in a communitydwelling Brazilian population. Alzheimer Dis Assoc Disord. 2004;18(4):241-6.

34. Perez F, Helmer C, Dartigues JF, Auriacombe S, Tison F. A 15-year population-based cohort study of the incidence of Parkinson's disease and dementia with Lewy bodies in an elderly French cohort. J Neurol Neurosurg Psychiatry. 2010;81 (7):742-6.

35. Rahkonen $\mathrm{T}$, Eloniemi-Sulkava $\mathrm{U}$, Rissanen $\mathrm{S}$, Vatanen $\mathrm{A}$, Viramo P, Sulkava R. Dementia with Lewy bodies according to the consensus criteria in a general population aged 75 years or older. J Neurol Neurosurg Psychiatry. 2003;74(6):720-4.

36. Rosenblatt A, Samus QM, Steele CD, Baker AS, Harper MG, Brandt J, et al. The Maryland Assisted-Living Study: prevalence, recognition, and treatment of dementia and other psychiatric disorders in the assisted-living population of central Maryland. J Am Geriatr Soc. 2004;52(10):1618-25.

37. Spada RS, Stella G, Calabrese S, Bosco P, Anello G, GueantRodriguez R-M, et al. Prevalence of dementia in a mountainous village of Sicily. J Neurol Sci. 2009;283(1-2):62-5.

38. Stevens T, Livingston G, Kitchen G, Manela M, Walker Z, Katona C. Islington study of dementia subtypes in the community. Br J Psychiatry. 2002;180:270-6.

39. Wada-Isoe K, Uemura Y, Suto Y, Doi K, Imamura K, Hayashi A, et al. Prevalence of dementia in the rural island town of Ama-cho, Japan. Neuroepidemiology. 2009;32(2):101-6.

40. Yamada T, Hattori H, Miura A, Tanabe M, Yamori Y. Prevalence of Alzheimer's disease, vascular dementia and dementia with Lewy bodies in a Japanese population. Psychiatry Clin Neurosci. 2001;55(1):21-5.

41. American Psychiatric Association. Diagnostic and Statistical Manual of Mental Disorders, 3rd ed. rev. Washington, DC: American Psychiatric Association; 1987.

42. American Psychiatric Association. Diagnostic and Statistical Manual of Mental Disorders, 4th ed. Washington, DC: American Psychiatric Association; 1994.

43. Hughes CP, Berg L, Danziger WL, Coben LA, Martin RL. A new clinical scale for the staging of dementia. $\mathrm{Br} \mathrm{J}$ Psychiatry. 1982;140:566-72.

44. World Health Organization. Tenth Revision of the International Classification of Diseases and Related Health Problems (ICD-10). Geneva: World Health Organization; 1992.

45. Cummings JL, Mega M, Gray K, Rosenberg-Thompson S, Carusi DA, Gornbein J. The Neuropsychiatric Inventory: comprehensive assessment of psychopathology in dementia. Neurology. 1994;44(12):2308-14.

46. Emre M, Aarsland D, Brown R, Burn DJ, Duyckaerts C, Mizuno Y, et al. Clinical diagnostic criteria for dementia associated with Parkinson's disease. Mov Disord. 2007;22(12):1689-707; quiz 837. 
47. Aarsland D, Zaccai J, Brayne C. A systematic review of prevalence studies of dementia in Parkinson's disease. Mov Disord. 2005; 20(10):1255-63.

48. Erkinjuntti T, Ostbye T, Steenhuis R, Hachinski V. The effect of different diagnostic criteria on the prevalence of dementia. N Engl J Med. 1997;337(23):1667-74.

49. Nelson PT, Jicha GA, Kryscio RJ, Abner EL, Schmitt FA, Cooper G, et al. Low sensitivity in clinical diagnoses of dementia with Lewy bodies. J Neurol. 2010;257(3):359-66.

50. Barker RA, Foltynie T. How common is dementia with Lewy bodies? J Neurol Neurosurg Psychiatry. 2003;74(6): 697-8.
51. Louis ED, Bennett DA. Mild Parkinsonian signs: an overview of an emerging concept. Mov Disord. 2007;22(12):1681-8.

52. Lopez OL, Hamilton RL, Becker JT, Wisniewski S, Kaufer DI, DeKosky ST. Severity of cognitive impairment and the clinical diagnosis of AD with Lewy bodies. Neurology. 2000;54(9): 1780-7.

53. Tsuang D, Simpson K, Larson EB, Peskind E, Kukull W, Bowen JB, et al. Predicting Lewy body pathology in a community-based sample with clinical diagnosis of Alzheimer's disease. J Geriatr Psychiatry Neurol. 2006;19(4):195-201.

54. Sinha N, Firbank M, O'Brien JT. Biomarkers in dementia with Lewy bodies: a review. Int J Geriatr Psychiatry. 2012;27(5):443-53. 